\title{
El lenguaje político en Twitter durante la segunda vuelta presidencial Colombia $2018^{*}$
}

\author{
Sandra Ximena Gallego Galvis** \\ Delsar Roberto Gayón Tavera*** \\ Juan Felipe Alzate Pongutá**** \\ Recibido: 2020-09-02 • Enviado a pares: 2020-09-20 \\ Aprobado por pares: 2020-11-05 • Aceptado: 2021-01-16 \\ https://doi.org/10.22395/angr.v20n39a5
}

\begin{abstract}
Resumen
La segunda vuelta electoral por la presidencia de Colombia, 28 de mayo al 16 de junio de 2018, se caracterizó por la ausencia de un debate entre los candidatos de la Colombia Humana y el Centro Democrático. La contienda electoral se centró en las redes sociales y los dos candidatos usaron, a través de la red social Twitter, estrategias del lenguaje de oposición, resistencia, encubrimiento, legitimación y deslegitimación que agudizaron la polarización entre el llamado al nacionalismo, la salvaguarda de las instituciones y un nuevo proyecto de país. El presente artículo tiene como objetivo evidenciar estas estrategias utilizadas por los aspirantes a la presidencia, durante la segunda vuelta electoral que circularon en Twitter con la finalidad de captar potenciales electores.

La investigación utiliza una metodología mixta compuesta de dos enfoques. El análisis descriptivo de corte cuantitativo para recuperar los 391 trinos emitidos desde las cuentas de Twitter de la Colombia Humana y el Centro Democrático durante las tres semanas de campaña. Posteriormente se efectuó un análisis crítico del discurso político para establecer la articulación de las relaciones entre política, redes sociales y lenguaje. Los hallazgos indican una continuidad en las prácticas políticas en relación con los dos anteriores periodos electorales caracterizados por el enfrentamiento y la hostilidad entre dos bloques ideológicamente opuestos: derecha y la izquierda.

Palabras clave: comunicación política, elecciones, partido político, redes sociales.
\end{abstract}

\footnotetext{
- Este artículo es resultado del proyecto de investigación Estrategias discursivas en redes sociales de los candidatos presidenciales durante la segunda vuelta electoral, Colombia 2018 (2019), adscrito al grupo de investigación Comunicación, Paz y Conflicto de la Facultad de Comunicación Social de la Universidad Santo Tomás sede Bogotá, Colombia.

" Doctora en Ciencias Humanas y Sociales, Universidad Nacional de Colombia, docente de la Facultad de Comunicación Social de la Universidad Santo Tomás, Bogotá. Email: sandragallego@usantotomas.edu.co Orcid: https://orcid.org/0000-0002-6649-4221

... Estudiante de Doctorado en Teoría Crítica en diecisiete, Instituto de Estudios Críticos Ciudad de México, México. Docente de la Facultad de Comunicación Social de la Universidad Santo Tomás, Bogotá. Email: delsargayon@usantotomas.edu.co Orcid: https:// orcid.org/0000-0002-4805-8565

.... Magíster en Filosofía de la Pontificia Universidad Javeriana, docente de la Facultad de Comunicación Social de la Universidad Santo Tomás, Bogotá, Email: juan.alzate@usantotomas.edu.co Orcid: https://orcid.org/0000-0003-3834-8822
} 


\title{
Political Language on Twitter during the Runoff Voting in the 2018 Presidential Election
}

\begin{abstract}
The second round of elections for the presidency of Colombia, May 28th-June 16th, 2018, was characterized by the absence of a debate between Humane Colombia and the Democratic Center candidates. The electoral contest focused on social networks and the two candidates used, through the Twitter social network, language strategies of opposition, resistance, cover-up, legitimization, and delegitimization that sharpened the polarization between the call to nationalism, the safeguarding of the institutions, and a new country project. This article aims to demonstrate these strategies used by presidential candidates during the second round of elections that circulated on Twitter to capture potential voters.

The research uses a mixed methodology composed of two approaches. The descriptive analysis of quantitative type to recover the 391 trills issued from the Twitter accounts of Humane Colombia and the Democratic Center during the three weeks of the campaign. Subsequently, a critical analysis of the political discourse was carried out to establish the articulation of the relations between politics, social networks, and language. The findings indicate continuity in political practices concerning the two previous electoral periods characterized by confrontation and hostility between two ideologically opposed blocs: right and left.
\end{abstract}

Keywords: political communication, elections, political party, social networks.

\section{A linguagem política no Twitter durante o segundo turno das eleições presidenciais na Colômbia no ano de 2018}

\begin{abstract}
Resumo
O segundo turno eleitoral para a presidência da Colômbia, que foi de 28 de maio ao 16 de junho de 2018, caracterizou-se pela ausência de um debate entre os candidatos da Colômbia Humana e o Centro Democrático. A disputa eleitoral centrou-se nas redes sociais e os dois candidatos usaram, através do Twitter, estratégias de linguagem de oposição, resistência, encobrimento, legitimação e deslegitimação que agravaram a polarização entre o nacionalismo, a salvaguarda das instituições e um novo projeto de país. O presente artigo tem como objetivo evidenciar ditas estratégias utilizadas pelos candidatos à presidência, durante o segundo turno eleitoral que circularam no Twitter com a finalidade de captar potenciais eleitores.
\end{abstract}

A investigação utilizou uma metodologia mista, composta de dois enfoques. A análise descritiva do tipo quantitativo para recuperar os 391 trinos emitidos a partir das contas de Twitter da coligação Colômbia Humana e do Centro Democrático, durante as três semanas de campanha. Posteriormente foi realizada uma análises crítica do discurso político para estabelecer a articulação das relações entre política, redes sociais e linguagem. Os resultados indicam uma continuidade nas práticas políticas em relação com as dos dois anteriores períodos eleitorais caracterizados pelo enfrentamento e a hostilidade entre dois blocos ideológicos opostos: direita e esquerda.

Palavras chave: comunicação política, eleições, partido político, redes sociais. 


\section{Introducción}

En el contexto actual de la comunicación política es posible afirmar que son extensos los estudios sobre las campañas electorales en las redes sociales (Rodríguez y Ureña, 2011). Dichos estudios pueden organizarse desde tres cuestionamientos centrales: primero, ¿cómo se desenvuelven las campañas en las redes?, segundo ¿cómo los candidatos se involucran en la comunicación en redes? y tercero ¿cómo interactúan con el público y entre ellos? (Stier et al., 2018 p. 52). Las investigaciones concuerdan en afirmar que es a partir de la campaña presidencial de Barack Obama en el 2008 que las redes sociales, mediatizadas por la tecnología digital, entran a ser una herramienta exitosa de comunicación política (López-Londoño, 2018; Zugasti y Sabés, 2015; Alonso-Muñoz y Casero-Ripollés, 2018; Vallejo, 2018). Redes como Twitter, Facebook, Instagram y Snapchat ofrecen patrones representativos de comportamiento y su uso puede tener influencia directa en los electores (Bossetta, 2018; Kreiss y Mcgregor, 2018).

El trabajo de investigación de Jungherr (2016) evidencia la prominencia de la red social Twitter en los análisis sobre campañas electorales. Esta revisión identifica ciento veinticiete investigaciones sobre el uso de Twitter en campañas electorales en diversos países. Dicho análisis agrupa las investigaciones en tres: el uso de Twitter por candidatos y partidos, el uso de Twitter por el público con vocería política y el uso de Twitter durante un evento mediático o en reacción a este. Si bien el trabajo en mención comunica resultados en cuanto al uso de Twitter por el público y el análisis cuantitativo del número de interacciones que genera cada mensaje, no realiza un análisis del uso estratégico del lenguaje inherente a este evento mediático.

El artículo "Barcelona En Comú on Twitter" plantea la discusión con base en los estudios sobre la comunicación política de esta red social durante las campañas electorales en España, y afirma que las comparaciones son útiles para entender las condiciones políticas y mediáticas de cada país entre candidatos, partidos y los discursos que estratégicamente ponen en circulación (García-Carretero y Pérez-Altable, 2017 p. 873). En estos estudios comparativos se encuentran varias posturas que pueden ser divididas en dos grandes grupos y que interesan a la presente investigación: las que identifican a Twitter como un espacio de comunicación dialógica y participativa (Carrasco Polaino, Villar Cirujano y Tejedor Fuentes, 2018 p. 67; Domínguez, 2015) y aquellas que ven a la red social como una herramienta utilizada por políticos y partidos para la reproducción de las lógicas de comunicación tradicionales (Graham, Jackson y Broersma, 2016 p. 767; Ramos-Serrano, 2018).

En la revisión de la literatura sobre el objeto de estudio, también se encuentran posiciones críticas como la de Boyadjian (2016), que afirma que se trata de información útil para extraer opiniones políticas, pero coincide con Severo y Lamarche-Perrin 
(2011) en que los "Tweets políticos", siguen siendo poco conocidos por los investigadores en cuanto a sus condiciones de configuración y las relaciones pragmáticas con usuarios de esta red social.

Estudios como los de Campos-Domínguez (2017) son un punto de inflexión a la crítica anterior porque apuestan por el análisis de los mensajes de los emisores y la función de Twitter en las campañas políticas. Dicho análisis contribuye "a relacionar las características textuales con las situaciones sociales concretas en que se producen y consumen los textos (...) las prácticas discursivas se entienden como mediadores entre los textos producidos institucionalmente y las prácticas socioculturales mucho más extensas" (Schroder, 2014, p. 185). Estas líneas de trabajo enfatizan en las relaciones entre lenguaje, política y tecnología y por lo tanto permiten una mirada crítica a los usos de la comunicación en situaciones concretas. Paveaum (2012), ofrece una mirada integradora en este campo, al llevar a cabo una crítica a la distinción entre lenguaje y técnica, para proponer un continuo e incluso un "ensamblaje" entre los dos para hablar de universos discursivos digitales: universos de discurso en línea totalmente integrados en las modalidades de habla e intercambio, que son aumentados por la tecnología.

Los estudios de Chilton y Schaffner (2005) son un aporte a estas miradas por cuanto se ocupan del uso político del lenguaje en los juegos de poder y/o de resistencia desde las "funciones estratégicas" del lenguaje entre las comunidades o grupos políticos que resultan más evidentes en las campañas electorales, que formulan sus estrategias discursivas acorde con las narrativas propias de los dispositivos tecnológicos vigentes.

El presente trabajo de investigación se enmarca en estas tendencias analizando las estrategias del discurso político en los niveles lingüísticos de los candidatos de los partidos la Colombia Humana y Centro Democrático durante la segunda vuelta electoral en las elecciones presidenciales 2018.

\section{Metodología}

En la mayoría de las investigaciones de Twitter en las campañas electorales se incluye una metodología mixta compuesta por enfoques cuantitativos y cualitativos (Jungherr, 2016). El análisis cuantitativo de corte descriptivo permitió trabajar con el universo de trinos emitidos desde las cuentas de Twitter de la Colombia Humana y el Centro Democrático durante las tres semanas de campaña. La recolección se realizó mediante un programa gestor de referencias bibliográficas con el que fueron descargados los trinos y posteriormente compilados en una base de datos en la cual se diferenció el número de trinos emitidos por cada partido.

En la siguiente fase se ordenaron los tuits sistemáticamente por partido político. Con este resultado, se realizó una medición de la información recolectada desde tres 
criterios: los más likeados, los más comentados y los más retuiteados. Este enfoque también permitió comparar el número de tuits emitidos por las cuentas de los dos partidos y establecer una frecuencia de emisión de Twitter entre el 28 de mayo y el 16 de junio.

En el enfoque cualitativo se realizó un análisis crítico del discurso político Chilton y Schaffner (2005) del universo de los trinos emitidos. Se elaboró una matriz de análisis con cuatro funciones estratégicas del lenguaje propuestas por estos autores:

\section{Coerción}

Se refiere a los actos de habla que contemplan lenguaje sancionatorio, actos jurídicos, prioridad en temas o asuntos, colocarse en relaciones específicas, realizar diagnósticos sociales, políticos, económicos y/o tipos de censura en el habla.

\section{Resistencia, oposición y protesta}

Se refiere a los medios de difusión (grafiti, afiches, vallas, entre otros) y a las expresiones lingüísticas (eslóganes, cánticos, petitorios, entre otras) adoptados por quienes se consideran opositores al poder dominante.

\section{Encubrimiento}

Se refiere al control político de la información como el ocultamiento, economizar la verdad, uso de evasivas y mentiras, formas de negación, omisión, uso de eufemismos y desvío de la atención.

\section{Legitimación y deslegitimación}

Se refiere a los usos del lenguaje caracterizados por la presentación positiva de sí mismo: liderazgo, carisma, auto adulación, autopresentación positiva de sí misma, alarde, deseo de los electores y principios ideológicos y por la presentación negativa del otro: estereotipos, invasores, acusaciones e insultos y oposición institucional.

\section{Resultados}

\section{Análisis cuantitativo}

El establecimiento de la base de datos permitió la definición de un universo total de trescientos noventa y un trinos de los dos partidos, de los cuales trescientos once fueron publicados en la cuenta de la Colombia Humana y ochenta pertenecientes a la cuenta de Twitter del Centro Democrático. 
La primera medición muestra una mayor actividad en Twitter de la Colombia Humana con un 80 \% del total de los tuits emitidos, frente a un $20 \%$ identificado en la cuenta del partido del Centro Democrático (ver tabla 1).

Tabla 1. Porcentaje de trinos por partido

\begin{tabular}{|c|c|c|}
\hline \multirow{2}{*}{$\frac{\text { Periodo }}{\text { Cuenta }}$} & \multicolumn{2}{|c|}{ Noviembre $28 / 2018$ - junio $16 / 2018$} \\
\hline & @CeDemocratico & @ColombiaHumana \\
\hline Total, de Twittees & 80 & 311 \\
\hline Porcentaje & $20,5 \%$ & $79,5 \%$ \\
\hline
\end{tabular}

Fuente: elaboración propia.

Otro resultado cuantitativo es la frecuencia de emisiones de mensajes por día en cada una de las cuentas de los partidos políticos. La tabla 2 evidencia que la Colombia Humana en los veinte días trinó en promedio quince veces diarias. Al graficar estos resultados, se identifican tres picos durante este periodo: el primero, entre el 7 y el 10 de junio, con cincuenta y cuatro trinos. El segundo, entre el 14 y el 15 con cincuenta y dos trinos. El tercero, entre el 11 y el 13, con veintinueve trinos (ver tabla 2 y figura 1).

Tabla 2. Frecuencia de trinos emitidos por día desdela cuenta@ColombiaHumana en el periodo mayo 28 a junio 16

\begin{tabular}{cc}
\hline Fecha & Trinos \\
\hline Mayo 28 & 6 \\
\hline Mayo 29 & 9 \\
\hline Mayo 30 & 4 \\
\hline Mayo 31 & 2 \\
\hline Junio 1 & 6 \\
\hline Junio 2 & 20 \\
\hline Junio 3 & 17 \\
\hline Junio 4 & 10 \\
\hline Junio 5 & 19 \\
\hline Junio 6 & 18 \\
\hline
\end{tabular}

\begin{tabular}{cc}
\hline Fecha & Trinos \\
\hline Junio 7 & 8 \\
\hline Junio 8 & 18 \\
\hline Junio 9 & 27 \\
\hline Junio 10 & 1 \\
\hline Junio 11 & 19 \\
\hline Junio 12 & 47 \\
\hline Junio 13 & 21 \\
\hline Junio 14 & 29 \\
\hline Junio 15 & 23 \\
\hline Junio 16 & 7 \\
\hline
\end{tabular}

Fuente: elaboración propia. 


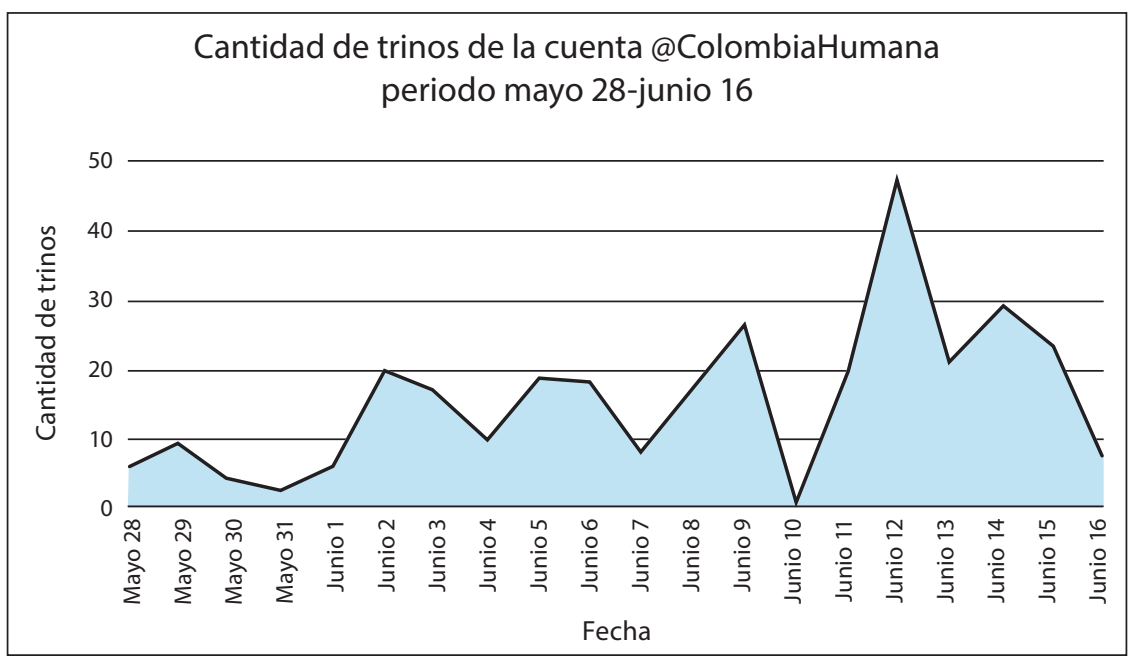

Figura 1. Frecuencia de trinos de la Colombia Humana durante la segunda vuelta Fuente: elaboración propia.

Por su parte, el Centro Democrático (ver tabla 3 y figura 2) trinó ochenta veces durante los veinte días para un promedio de cuatro trinos diarios. Pese al bajo número de trinos, también se identifican tres picos entre el 8 y el 16 de junio, coincidiendo con las fechas de los picos alcanzados por la Colombia Humana. El primer pico del Centro Democrático es entre el 7 y el 9 de junio con un total de dieciocho trinos. El segundo pico se encuentra entre el 10 y el 12 de junio con un total de quince trinos. El último pico está entre el 13 y el 14 de junio con un total de catorce trinos.

Tabla 3. Frecuencia de trinos emitidos por día desdelacuenta@CeDemocratico en el periodo mayo 28 a junio 16

\begin{tabular}{cc}
\hline Fecha & Trinos \\
\hline Mayo 28 & 2 \\
\hline Mayo 29 & 2 \\
\hline Mayo 30 & 1 \\
\hline Junio 3 & 3 \\
\hline Junio 4 & 4 \\
\hline Junio 5 & 7 \\
\hline Junio 6 & 4 \\
\hline Junio 7 & 3 \\
\hline
\end{tabular}

\begin{tabular}{cl}
\hline Junio 8 & 4 \\
\hline Junio 9 & 11 \\
\hline Junio 10 & 3 \\
\hline Junio 11 & 9 \\
\hline Junio 12 12 & 3 \\
\hline Junio 13 & 5 \\
\hline Junio 14 15 & 9 \\
\hline Junio 15 & 6 \\
\hline Junio 16 & 4 \\
\hline
\end{tabular}

Fuente: elaboración propia. 


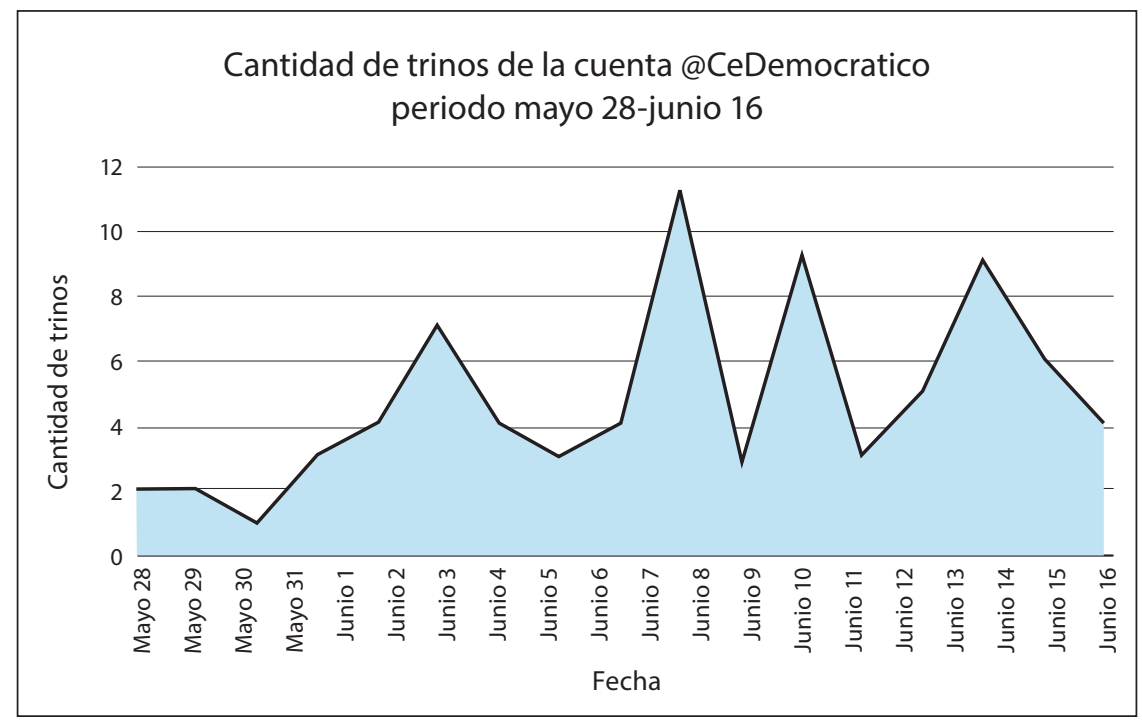

Figura 2. Frecuencia de trinos del Centro Democrático durante la segunda vuelta. Fuente: elaboración propia.

La gráfica 3 permite visualizar el comparativo por partido de la actividad de Twitter durante los veinte días de campaña por la segunda vuelta electoral.

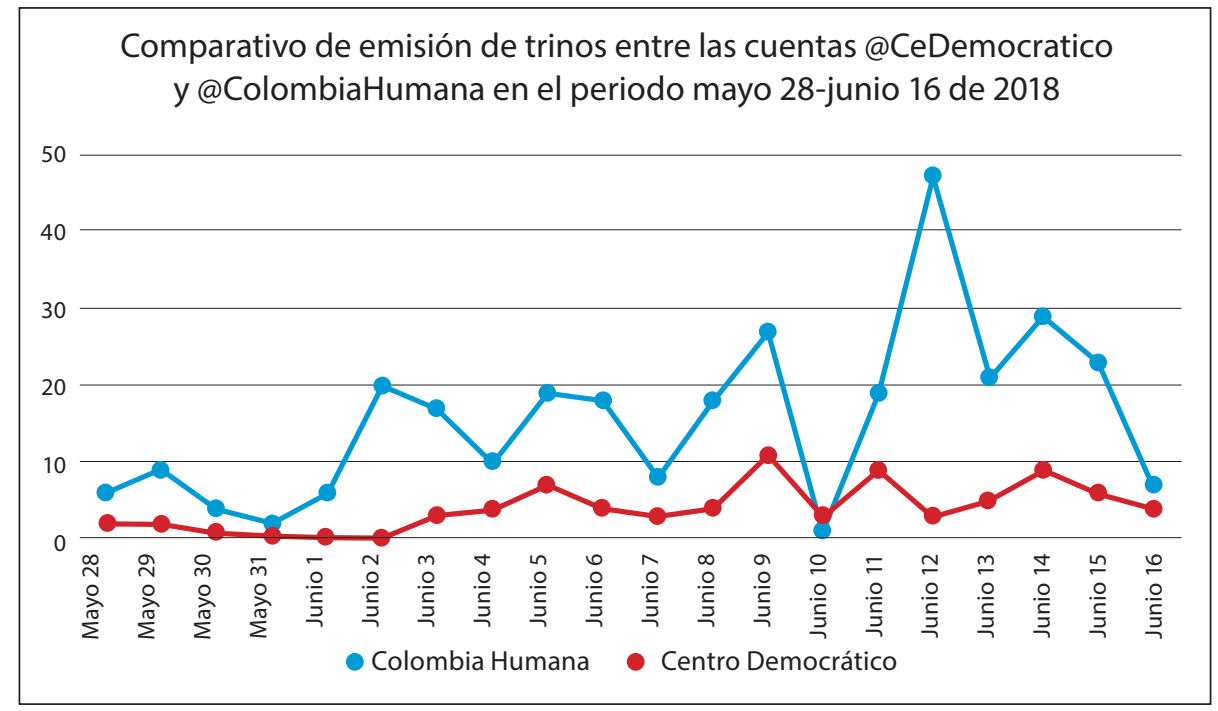

Figura 3. Comparativos de frecuencia de trinos durante la segunda vuelta Fuente: elaboración propia 


\section{Análisis cualitativo: usos estratégicos del lenguaje político}

\section{Estrategia de coerción}

Se identificó como primer uso estratégico del lenguaje en la cuenta de la Colombia Humana, la coerción en la cual el partido se asume como una autoridad política para enunciar, a través de un lenguaje sancionatorio y de desaprobación, las acciones de las fuerzas políticas opositoras, en una clara recurrencia a los actos de habla de carácter expresivo, sobre todo por las reiteradas acusaciones de culpabilidad.

Los tuits se refieren a los casos más representativos en Colombia sobre corrupción, fraude electoral, robos, sobornos, parapolítica, censura y violación a los derechos constitucionales. Otras publicaciones aluden directamente al contendor político, Iván Duque, para cuestionar públicamente el apoyo de seguidores como los clanes políticos de la Costa Atlántica de Colombia. Dos tuits ilustrativos al respecto son los de la Colombia Humana (2018, 15 de junio): "\#DuqueEsCorrupción cuando permite que una persona condenada por robar el alimento de los niños en la Guajira le haga campaña" y "\#DuqueEsCorrupción en el César nos reportan que los siguientes clanes están trabajando en la campaña de @IvanDuque: Clan Gneco: parapolítica y corrupción. Didier Lobo: Corrupción. Ape Cuello: parapolítica y corrupción. Clan Araujo: Parapolítica y Corrupción".

A partir del 7 de junio, la Colombia Humana cambió el discurso de coerción de un tono de denuncia hacia la deliberación política. Publicó doce tuits reclamando como derecho ciudadano el debate público de los candidatos presidenciales. En estas publicaciones se interpelan a organismos del Estado como la Corte Constitucional y el Consejo Nacional Electoral, y se hace un llamado a su competidor a respetar el debate democrático de las ideas. En general, se encontró un uso extensivo de actos de habla directivos (órdenes) con llamados permanentes a respetar los derechos y la democracia, y comisivos (promesas) sobre los derechos a la información. Así mismo, se instó al sistema público y privado de medios en Colombia para que, mediante la generación del debate, se garantizara un pluralismo informativo. Se destaca como el tuit más representativo el de la Colombia Humana (2018, 12 de junio) "\#DuqueTemeAlDebate ¿Les negará a los ciudadanos su derecho a elegir según el libre debate de las ideas?"

Este partido también hizo un uso estratégico del lenguaje buscando establecer relaciones de vínculo a su propuesta política y de oposición a los poderes hegemónicos en Colombia. En las relaciones de vínculo e integración se hace un llamado a los actores de la sociedad civil, entre los cuales se cita la organización de trabajadores, las clases sociales, las familias, las madres de Colombia, los jóvenes, la clase media rural y las etnias. La estrategia propone la construcción de un capitalismo basado en la modernización del campo, la justicia social, la decencia y la seguridad ciudadana. 
La denuncia generalizada de oposición y rechazo apunta al entrampamiento entre el Estado y los latifundistas. Para este argumento se acude a los actos de habla expresivos, tales como el uso de palabras como fraude, dictadura, violencia, desigualdad y falta de procesos democráticos en Colombia. El siguiente tuit sintetiza este tipo de relación: Colombia Humana (2018, 12 de junio) "@petrogustavo con sindicatos: La mayoría de los capitalistas en Colombia son empresarios de garaje acosados por los impuestos y la mafia porque el Estado está al servicio del latifundio"

Por su parte, el Centro Democrático se apoyó en actos de habla comisivos a través del manejo de las emociones. En términos de coerción, orientó el uso estratégico del discurso hacia tres tipos de potenciales electores: la ciudadanía en general, los miembros de partidos políticos junto con las organizaciones gremiales y sindicales, y los trabajadores de la cultura. La estrategia discursiva para la ciudadanía se evidenció al hablar a nombre de todos los colombianos (la frecuencia de ocurrencia del plural nos resulta significativa para incluir a los destinatarios en el discurso), a través de tuits que prometen acceso a oportunidades de trabajo y que demandan participación ciudadana en los asuntos públicos. Este uso del nosotros discursivo se combina con el manejo de emociones y sentimientos: expresiones de afecto y respeto, muestras de gratitud y cariño, recuperación del honor y mantenimiento de la alegría y el entusiasmo.

En esta misma línea de establecer relaciones de vínculo (que presuponen legitimidad por parte del partido y hacia el partido), el Centro Democrático se dirige a comunidades específicas convocando y agradeciendo su rol de voluntarios para el apoyo y difusión de las propuestas de campaña. El discurso articuló tres dimensiones: geográfica, que intenta dar cuenta de la extensión territorial del país, nombrando ciudades capitales como Medellín, Villavicencio, Cali, Bogotá y municipios como San Jacinto, Cotorra, Tocancipá, Girardota, Villa de Leyva y las localidades de Usaquén y Kennedy. La social, para dar un reconocimiento a los distintos actores, buscando fortalecer las relaciones líder-liderados: jóvenes emprendedores, voluntarios rurales, trabajadores del sector de la salud, víctimas y agricultores, así como el Consejo Gremial Nacional y la Sociedad de Agricultores de Colombia (SAC). Por último, con menor cantidad de trinos, una dimensión ética enmarcada por expresiones de gratitud, relaciones hacia los artistas del Vallenato, al sector del entretenimiento y a los deportistas que se adhirieron a la propuesta política de Iván Duque y reconocen que sus promesas de campaña son un vehículo de cohesión social. En este sentido, se encuentra en los usos del lenguaje del Centro Democrático un intento por construir un líder y por fabricar consensos al interior de su partido y con sus simpatizantes.

En esta misma línea estratégica, los vínculos de cohesión para abrir canales de comunicación se evidenciaron a través de la utilización de las redes sociales análogas y digitales con invitaciones a la gente a participar en Facebook Live y Twitter para 
interactuar, dialogar y despejar dudas. Se identifican dos mensajes que pusieron al Centro Democrático en desacuerdo con las Farc y con el Gobierno en el proceso de nombrar contralor de la República.

En general, el nivel discursivo de la cuenta de Twitter del Centro Democrático se apoyó en actos de habla comisivos para mantener viva la esperanza de que los problemas cruciales del país serán resueltos.

\section{Estrategia de resistencia, oposición y protesta}

Los juegos estratégicos del lenguaje político que manifestaron resistencia, oposición y protesta estuvieron presentes en los tuits de las dos campañas, pero con un mayor número de trinos de la Colombia Humana. Estos discursos estratégicos ponen en juego dos dimensiones principales: ética y política.

En el caso de la Colombia Humana, la dimensión ética cuando se refiere a su propio proyecto político, se identifica una estrategia relacionada con los campos semánticos del sentimiento y la acción, a través de tuits que invitan a construir un estado de vida futuro con un lenguaje emotivo para los ciudadanos, pero sin enunciar directamente cómo se accederá a dicho estado. Además, se posiciona apelando a un sentido optimista de la vida, los sueños, la esperanza, la decencia, la limpieza, la austeridad, la alegría y el respeto. Por el contrario, cuando se refiere al proyecto de su oponente hace juicios morales, cuestionando la trayectoria de la vida pública de algunos de sus miembros y recordándoles los procesos jurídicos en su contra.

Por otra parte, el discurso de la Colombia Humana, a través de su cuenta de Twitter, denuncia una violación a los códigos éticos de la libertad de expresión por parte de los medios tradicionales de Colombia. Este partido usa un nivel pragmático de expresión para acusar a estos medios masivos de comunicación como carentes de objetividad e imparcialidad frente al tratamiento de la información que da el partido Centro Democrático. Por ejemplo, cuando los medios omiten cuestionamientos éticos sobre supuestos vínculos ilegales de la familia Uribe. Así mismo, los trinos niegan y denuncian la falta de profesionalismo de los medios frente al manejo de los resultados de las encuestas de opinión.

En la dimensión política, el juego del lenguaje se muestra a favor del cambio y la construcción de un nuevo Gobierno desde las diferencias, la inclusión, la paz, la libertad, la democracia y el progresismo. Usa metáforas del juego como fórmulas ganadoras, jugar unidos para ganar, con toda para ganar, jugar los últimos tres minutos del partido como los tres primeros y no caer en la opción de votar en blanco porque: "Hoy el voto en Blanco lleva a la victoria a Duque. Mi invitación es a decidir. Yo estoy convencido de que el voto en blanco se decidirá por Petro y ganaremos". Esto permite 
derrotar en las urnas a "los de siempre" en clara alusión a las élites políticas que han gobernado a Colombia (2018, 6 de junio).

La Colombia Humana denuncia que es objeto de mentiras y difamaciones por parte de sus contradictores políticos y mediáticos a través del rechazo del proyecto político en el ámbito internacional, sobre todo en temas de calificación de riesgos e inversión, y en la aplicación del modelo venezolano en Colombia. Para ello, utilizan acusaciones de culpabilidad como comunista, ateo y extremista al proyecto de la Colombia Humana. Otra forma de respuesta a este macartismo es apoyarse en el discurso de otros actores del espectro político como la Misión de Observación Electoral (MOE) para señalar delitos como alteraciones al censo electoral y formularios de votación a favor de Iván Duque.

La Colombia Humana afirma que toda esta estrategia en su contra obedece a la falta de un proyecto político del Centro Democrático, razón por la cual se muestran adalides de la verdad acudiendo a principios católicos: "El mensaje de Jesús es el de la verdad, es el de la vida, es el de la luz. 'Yo soy el camino, la verdad y la vida', decía. Miren donde está la mentira, allá, en la otra campaña" (2018, 13 de junio) y al mismo tiempo, actuando por fuera del marco de la legalidad, se muestran como portadores de prácticas ético-políticas: Duque propone una campaña limpia ante las cámaras, pero pagan miles de millones de pesos en campaña negra contra@petrogustavo (2018, 14 de junio).

El análisis de la dimensión ética en las pocas publicaciones del Centro Democrático, reveló una prevalencia de los actos de habla expresivos con permanentes acusaciones y descalificaciones a su opositor. Es recurrente en los trinos acudir al valor de la palabra, la honra, la esperanza y la actitud positiva. Todos ellos, términos emotivos puestos en defensa del país frente al surgimiento de otras formas de organización sociopolítica en Colombia. La dimensión ética, en estrecha relación con la dimensión política, buscó sacar de la contienda electoral a su opositor a través del juego del encasillamiento en una orientación ideológica que este partido cuestiona como antidemocrática y fracasada en los modelos de desarrollo económico y social: "No podemos dejar el futuro de la Patria en manos del socialismo que tiene a la hermana Venezuela hundida en la miseria" (2018, 9 de junio). Hace parte del juego de este partido descalificar el programa de su oponente, al tiempo que posicionarse como víctima de información falsa: "Gerente de campaña de @IvanDuque denuncia información falsa en redes sociales" (2018, 16 de junio).

\section{Estrategias de legitimación y deslegitimación}

La mayor cantidad de trinos de las cuentas de la Colombia Humana en cuanto a las estrategias de "legitimación" fue la presentación positiva de sí mismo, mediante 
actos de habla expresivos que elogian al candidato y su proyecto político. En primer lugar, su defensa a un modelo económico antineoliberal, pero defendiendo la libre competencia y la propiedad privada a través de la democratización del capital y de la no expropiación. Los trinos hablan de favorecer la productividad de los campesinos y la clase media. Se encuentran tres temas en la agenda económica: el de las energías limpias y renovables contra el cambio climático, la medicina preventiva y el tema del emprendimiento para todos: banca pública, pequeños y medianos empresarios.

Es reiterativo en los trinos de este partido buscar el fortalecimiento de la clase media y rural en consonancia con las variables económicas mencionadas anteriormente. El siguiente trino muestra la consonancia con lo rural: "Tenemos tres aliados: el aguacate, la abeja obrera, la papaya, Toto la Momposina, sindicatos, iglesias (defendieron la libertad de culto) del extractivismo hacia una economía productiva (2018, 14 de junio)".

En segundo lugar, la estrategia de legitimación a través de actos lingüísticos declarativos que exaltan el respeto por el Estado Social de Derecho y la independencia entre los poderes que lo conforman. Se compromete a defender los derechos democráticos de los ciudadanos como la justicia social, la pluralidad, la transparencia electoral, el derecho a la vida digna, la educación, el saber y el conocimiento, la salud, la niñez, las personas en condición de discapacidad, la pluralidad cultural, entre otras muchas.

En tercer lugar, la Colombia Humana declara a la educación un eje central dentro de su modelo de desarrollo económico, pero considera importante la gratuidad y la ampliación del acceso a la educación superior. Las publicaciones expresan un cierto continuismo de los proyectos sociales de Gobiernos anteriores, tales como Familias en Acción, en función ahora de la productividad. La Colombia Humana, en su carrera por la presidencia, no se declaró como opción totalmente independiente. Es por eso que continuamente reiteró una alianza en pro de la reconciliación con diversos partidos políticos como el Polo Democrático y la Alianza Verde (apoyar un cambio con ética), los Decentes, los jóvenes fajardistas, y el Movimiento Alternativo Indígena y Social, pluralidad de cultos y propone un cambio con reconciliación y continuidad en la construcción de la paz, "en el que los ciudadanos podemos pensar distinto sin ser enemigos. Pastores y pastoras de la iglesia cristiana: se defiende la libertad de cultos desde la constitución de 1991" (2018, 8 de junio).

El análisis de lo anterior muestra una prevalencia de los actos de habla declarativos en su discurso político y en su insistencia por marcar públicamente las bases de su proyecto político. Muestra de ello es versatilidad de sus estrategias de legitimación. A continuación, se referencian de manera genérica otras complementarias a las ya mencionadas: 
- Alarde: apoyo que le brindan públicamente otros actores sociales a este partido, como lideresas de la diversidad, líderes de la transparencia en la gestión pública. Así como de los sindicatos, los nuevos movimientos culturales, ambientalistas y de derechos humanos. También, hace alarde del apoyo de personajes y figuras internacionales en el campo de la cultura y la academia como Slavoj Žižek y Thomas Piketty.

- Presunción de las capacidades políticas, intelectuales y comunicativas de Gustavo Petro.

- Invocación de personajes políticos que han significado cambios en la construcción de la Nación colombiana como Alfonso López Pumarejo, Jorge Eliecer Gaitán y Luis Carlos Galán y Álvaro Gómez Hurtado.

- Desencasillarse de las vertientes ideológicas con posiciones maniqueas entre derecha-centro-izquierda moderada violencia-paz, difamación-soluciones, legalidad-corrupción, expropiación-libre venta de la propiedad, corruptos-soberanía alimentaria; corrupción vs democracia plena, cambio vs politiquería.

- Enaltecimiento de Petro como ciudadano colombiano desde su ascendencia familiar rural trabajadora, con educación católica, estudiante de alto rendimiento y políticamente con opción para los pobres.

Las estrategias de deslegitimación de la Colombia Humana se relacionan con el uso estratégico de la descalificación de las acciones políticas del partido de la oposición, a través de actos de habla expresivos que los señalan de corruptas, oscurantistas, dictatoriales de la justicia y la existencia de una alianza entre narcotráfico, Estado y política. Otras se encuentran en el plano de la economía, asociando una continuidad de la política de Uribe, Pastrana y Gaviria, en cuanto abrir el país a las inversiones de las multinacionales en detrimento de la industria nacional.

Adicionalmente la Colombia Humana afirma que es deslegitimada públicamente desde una competencia política desleal, al acusar de populista a su candidato Gustavo Petro: "La campaña de @IvanDuque propone una campaña limpia ante las cámaras, pero pagan miles de millones de pesos en campaña negra contra@petrogustavo". Cuando no hay argumentos la estrategia es el ataque y el desprestigio \#ColombiaHumanaSePuede (2018, 28 de mayo).

Por su parte, el uso estratégico de la "legitimación del Centro Democrático", acude a actos de habla "declarativos" que apuntan a hacer una presentación positiva del candidato y del partido, señalando como puntos fuertes el respeto por el Estado Social de Derecho con independencia del poder de la justicia y la relación equilibrada entre el poder legislativo y judicial. No obstante, deja abierta la puerta a una reforma de la justicia. 
De igual manera, en los trinos se evidencia la intención de ampliación de la institucionalidad a través del Ministerio de deporte y la creación de un Banco Agrario que favorezca al pequeño productor. También incluye en su proyecto defender los derechos de la niñez, de las personas en condición de discapacidad y los adultos mayores y su compromiso con los acuerdos de paz: "reafirmó su compromiso con la reinserción de la base guerrillera" (2018, 15 de junio).

Otros trinos se comprometen con la ciudadanía en temas relacionados con la consulta anticorrupción, la despolitización de las instituciones del poder rural, ampliar la cobertura en salud del adulto mayor, y el desarrollo económico y social para las regiones. Se compromete, a través de los trinos, a conservar la vigencia de la acción de tutela como mecanismo de defensa de los derechos ciudadanos y le asigna a la fuerza pública la función de proteger la biodiversidad.

El alarde es otro uso estratégico del lenguaje en la cuenta de Twitter del Centro Democrático, que mayoritariamente, mediante actos de habla expresivos, se elogia la difusión de la propuesta política en diferentes regiones de Colombia. Se enfatiza en los continuos encuentros con la ciudadanía en las localidades y barrios de las principales ciudades como Bogotá, Medellín, Cali, Cundinamarca y Cúcuta, y también se presenta en las regiones fronterizas, Leticia y Putumayo. Antes que evidenciar el contenido de las propuestas, los trinos invitan a un patriotismo con expresiones de exaltación y alabanza como amor a la patria, tales como: "iVamos Colombia!", "Gratitud con la ciudadanía", "Nuestro futuro está en juego", "Construir país para todos", "Por el futuro de todos" o "Colombia es un país con una riqueza étnica inigualable".

En los dos partidos las estrategias de deslegitimación presentan un denominador común: realizan acusaciones mutuas sobre los modos en que se hacen las campañas. Así, campaña negra, cuando el Centro Democrático califica a su opositor; campaña limpia, cuando el Centro Democrático se autodefine políticamente; campaña burda, cuando la Colombia Humana define el accionar de su opositor. En la figura 4, se muestra de manera esquemática los usos estratégicos del lenguaje político en las campañas de la segunda vuelta presidencial, Colombia 2018.

\section{Discusión}

Los hallazgos sobre las estrategias discursivas en Twitter de los candidatos de la Colombia Humana y el Centro Democrático para persuadir votantes en la segunda vuelta de las elecciones presidenciales 2018, dialogan con los hallazgos de LópezLondoño (2018) en que la red recrea escenarios de enfrentamiento y hostilidad entre dos bloques ideológicamente opuestos y en los cuales los candidatos cuestionan los mensajes, las propuestas, las campañas y los perfiles morales de sus contendientes. Se difiere de la postura de Orhuela (2011), quien considera que en Twitter triunfa la cordialidad y el ingenio antes que la gresca. 


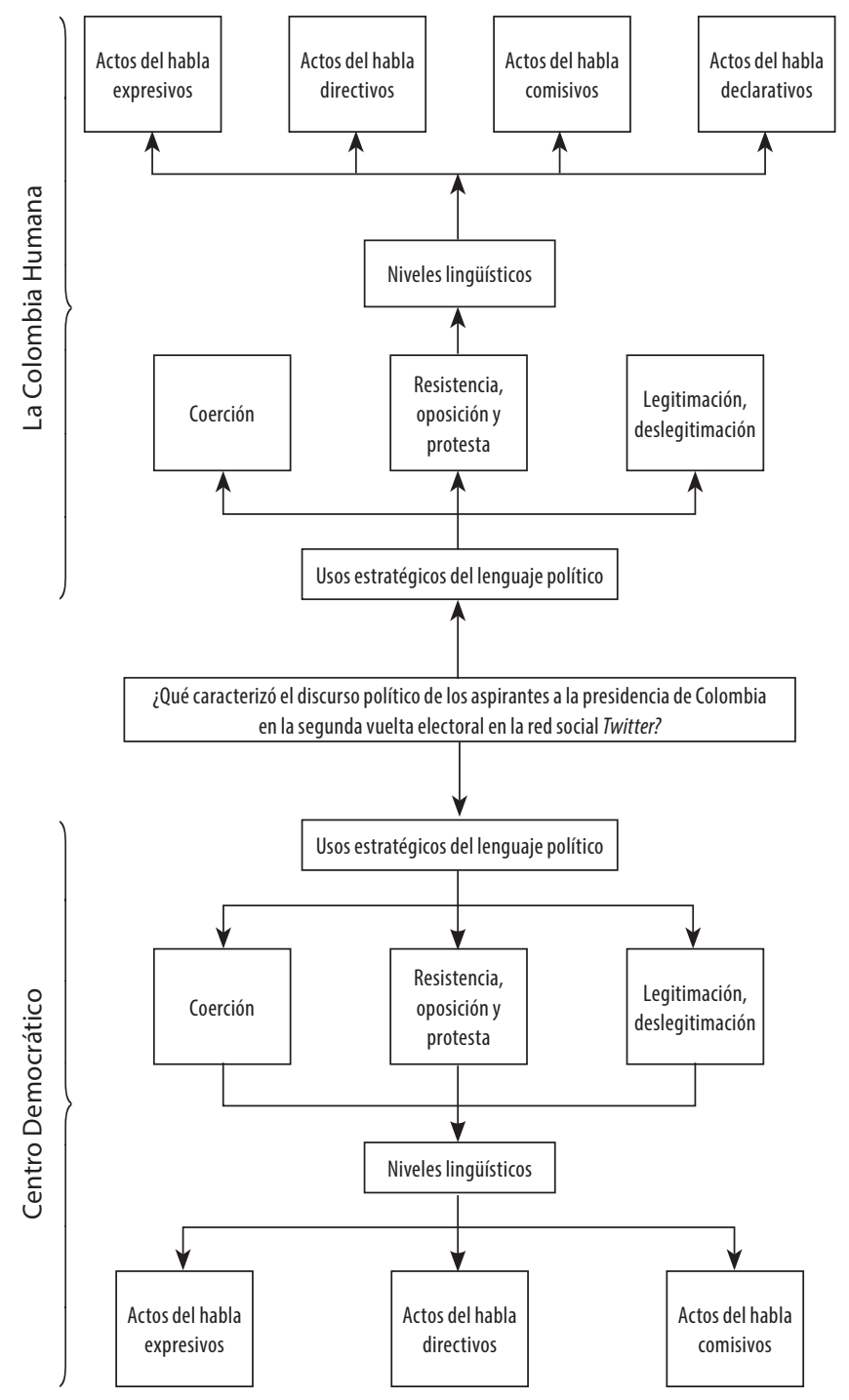

Figura 4. Usos estratégicos del lenguaje político

Fuente: elaboración propia.

Según Rieder (2012), la ironía y el humor son facilitadoras de las negociaciones no formales en Twitter, los hallazgos de la presente investigación muestran que esta red social es escenario directo de enfrentamientos y hostilidades. Esto se hizo evidente en la campaña de la Colombia Humana cuando deslegitimaba las acciones políticas del partido Centro Democrático, señalándolas de ilícitas, retrógradas, autoritarias y de alianzas con el crimen organizado. 
Los resultados de esta investigación coinciden con el análisis de tuits desde el punto de vista lingüístico, socio discursivo y socio pragmático que reconocen la prevalencia de estrategias, entendidas como formas de configurar mecanismos de poder y de reproducción de poder, tales como: criticar, denunciar, censurar, recriminar, legitimar-deslegitimar, conmover, adherir y buscar aceptación (Arrieta y Avendaño, 2018). En el caso del uso de Twitter en la segunda vuelta electoral por parte de los dos candidatos, primó la estrategia de legitimación basada en poner en evidencia su defensa del Estado Social de Derecho en Colombia: los tuits de la Colombia humana legitiman su candidato a través de un proyecto económico que apuesta por la democratización del capital a través del desarrollo de la clase media rural y de las energías limpias y renovables. El Centro Democrático coincide en la defensa del Estado social de Derecho con reformas y compromiso con los acuerdos de paz y una estrategia de encuentro permanente con la ciudadanía.

Respecto de la deslegitimación, la Colombia Humana se centró en cuestionar permanentemente el discurso y las actuaciones políticas del partido de la oposición, señalándolas de continuistas de las ideas políticas de la extrema derecha. Este mismo partido, denuncia una estigmatización de su campaña como populista, por parte del Centro Democrático, información que se evidencia en los tuits emitidos que se dedican a encasillarla ideológicamente como antidemocrática y fracasada.

Lo anterior confirma que el uso mayoritario de Twitter está en función del ataque de los políticos a sus contradictores (Cifuentes y Pino, 2018). En esta misma línea, los retuits de los congresistas del Centro Democrático se enfocan menos en sus propuestas o temas particulares de campaña y más en el uso de esta red para atacar a los antagonistas de su partido. También se basa en conseguir votantes mediante el manejo de las emociones, sentimientos y afectos, tales como el respeto, la gratitud, el cariño, la alegría y el entusiasmo, el valor de la palabra, la honra, la esperanza y la actitud positiva. En este aspecto se coincide con Slimovich (2016), cuando afirma que en las discursividades digitales de la comunicación política hay una mezcla de elementos de la argumentación racional con apelaciones a las pasiones de los internautas.

Finalmente, Guerrero-Solé y Mas-Manchón (2017) afirman que los partidos políticos no aprovechan el potencial dialógico de Twitter y apuestan por la unidireccionalidad, coincidiendo con las preocupaciones de Welp, Freidenberg y Capra (2018) sobre la contribución de los medios digitales a cerrar la brecha política entre la ciudadanía y los políticos. El hallazgo de la investigación muestra que los usos del lenguaje político de la Colombia Humana en su mayoría, envían mensajes unidireccionales a los ciudadanos cargados de información y con poca convocatoria al diálogo y al encuentro.

En la misma línea Roginsky y Cock, (2015) afirman que este dispositivo no aparece como el lugar de real diálogo entre representantes y representados. Por su parte, 
Longhi (2013) matiza esta posición e identifica el tuit político como un discurso en sí mismo que permite la constitución de un ethos discursivo. La anterior postura queda demostrada cuando el Centro Democrático anuncia e invita a los ciudadanos para que se conecten, participen, dialoguen y despejen dudas, es decir, que interactúen permanentemente sobre el candidato y su proyecto político.

De manera global se puede afirmar que los dos partidos políticos coinciden en hacer uso de las estrategias de lenguaje político en cuanto a coerción, resistencia, oposición-protesta y legitimación-deslegitimación. De acuerdo con la metodología planteada se identificó que estos dos partidos no utilizaron la estrategia de encubrimiento. En lo relacionado con los niveles lingüísticos en los tuits de la Colombia Humana y el Centro Democrático se encontró coincidencia en el uso de actos de habla declarativos y comisivos. Sin embargo, hay una recurrencia de la Colombia Humana a los actos de habla directivos para hacer un llamado al sistema de medios tradicionales de Colombia a respetar los principios democráticos de libertad de expresión y el debate público.

El análisis ratifica la tendencia de los últimos procesos electorales de polarización entre dos fuerzas políticas que tienen un proyecto distinto de país. En Twitter la construcción discursiva aleja cada vez más estos dos extremos y se empeñan en deslegitimarse mutuamente frente a la opinión pública.

\section{Referencias}

Alonso Muñoz, L. y Casero Ripollés, A. (2018). Political Agenda on Twitter During the 2016 Spanish Elections: Issues, Strategies, and Users' Responses. Communication \& Society, 31(3), 7-25.

Arrieta, L. y Avendaño, G. (2018). El discurso del tuit: un análisis lingüístico, sociodiscursivo y sociopragmático. Cuadernos de Lingüística Hispánica (32), 107-113. https://doi. org/10.19053/0121053X.n32.2018.8123

Bossetta, M. (2018). The Digital Architectures of social media: Comparing Political Campaigning on Facebook, Twitter, Instagram, and Snapchat in the 2016 U.S. Election. Journalism \& Mass Communication Quarterly, 95(2), 471-496. https://doi.org/10.1177/1077699018763307

Boyadjian, J. (2016). Les usages politiques différenciés de Twitter: Esquisse d'une typologie des "twittos politiques". Politiques de communication, 6(1), 31-58.

Campos Domínguez, E. (2017). Twitter y la comunicación política. El Profesional de la Información, 26(5), 785-793.

Carrasco Polaino, R., Villar Cirujano, E. y Tejedor Fuentes, L. (2018). Twitter como herramienta de comunicación política en el contexto del referéndum independentista catalán: asociaciones ciudadanas frente a instituciones públicas. Icono 14 Revista Científica de Comunicación y Tecnologías Emergentes, 16(1), 64-85. https://doi.org/10.7195/ril4.v16i1.1134 
Centro Democrático. (2018, 9 de junio). No podemos dejar el futuro de la Patria en manos del socialismo que tiene a la hermana Venezuela hundida en la miseria. [tuit]. https://twitter.com/ CeDemocratico/status/1005504905430388736.

Centro Democrático (2018,15 de junio). Reafirmó su compromiso con la reinserción de la base guerrillera. [tuit] https://twitter.com/CeDemocratico/status/1007642740383985666

Centro Democrático. (2018, 16 de junio).Gerente de campaña de@IvanDuque denuncia información falsa en redes sociales. [tuit] https://twitter.com/CeDemocratico/status/1008093791985852416

Chilton, P. y Schaffner, C. (2005). Discurso y política. El discurso como interacción social. Estudios sobre el discurso II. Una introducción multidisciplinaria. Gedisa.

Cifuentes, C. y Pino, J. (2018). Conmigo o contra mí: análisis de la concordancia y las estrategias temáticas del Centro Democrático en Twitter. Palabra Clave, 21(3), 885-916.

Colombia Humana. (2018, 28 de mayo). Cuando no hay argumentos la estrategia es el ataque y el desprestigio. [tuit]. https://twitter.com/ColombiaHumana_/status/1001293167570903040

Colombia Humana. (2018, 6 de junio). Hoy el voto en Blanco lleva a la victoria a Duque. Mi invitación es a decidir. Yo estoy convencido de que el voto en blanco se decidirá por Petro y ganaremos. [tuit]. https://twitter.com/ColombiaHumana_/status/1004366957188182016

Colombia Humana. (2018, 8 de junio). A un cambio con reconciliación en el que los ciudadanos podemos pensar distinto sin ser enemigos. [tuit]. https://twitter.com/ColombiaHumana_/ status/1005128305471877120

Colombia Humana (2018,12 de junio). La mafia porque el Estado está al servicio del latifundio. [tuit]. https://twitter.com/ColombiaHumana_/status/1006688524928765952

Colombia Humana. (2018, 12 junio). \#DuqueTemeAlDebate ¿Les negará a los ciudadanos su derecho a elegir según el libre debate de las ideas? [tuit]. Twitter. https://twitter.com/ ColombiaHumana_/status/1006560682895081472

Colombia Humana. (2018, 13 de junio). El mensaje de Jesús es el de la verdad, es el de la vida, es el de la luz. "Yo soy el camino, la verdad y la vida", decía. Miren donde está la mentira, allá, en la otra campaña. [tuit]. https://twitter.com/ColombiaHumana_/status/1006963182668271617

Colombia Humana. (2018, 13 de junio). Duque propone una campaña limpia ante las cámaras, pero pagan miles de millones de pesos en campaña negra contra @petrogustavo. [tuit]. https://twitter.com/ColombiaHumana_/status/1001293167570903040

Colombia Humana. (2018,14 de junio). Tenemos tres aliados: el aguacate, la abeja obrera y la papaya. [tuit]. https://twitter.com/ColombiaHumana_/status/1007315035406905345

Colombia Humana. (2018, 15 de junio). \#DuqueEsCorrupción cuando permite que una persona condenada por robar el alimento de los niños en la Guajira le haga campaña [imagen] [tuit]. Twitter. https://twitter. com/ColombiaHumana_/status/1007730138187030530

Colombia Humana. (2018, 15 de junio). \#DuqueEsCorrupción en el César nos reporta que los siguientes clanes están trabajando en la campaña de@IvanDuque Clan Gneco: parapolítica y corrupción Didier Lobo: 
Corrupción Ape Cuello: parapolítica y corrupción. Clan Araujo: Parapolítica y Corrupción [imagen] [tuit]. Twitter. https://twitter.com/ColombiaHumana_/status/1007736544806006785

Domínguez, N. (2015). Política 2.0. La comunicación política mediada por Twitter: ¿alternativa o complemento de los medios de comunicación masiva tradicionales? Estudio de la comunicación política y de sus repercusiones mediáticas centrado en la figura de la presidenta Cristina Fernández de Kirchner, en el período agosto-octubre de 2011 [tesis de doctorado, Universidad Nacional de la Plata]. http://hdl.handle.net/10915/46845

García Carretero, L., y Pérez Altable, L. (2017). Barcelona En Comú on Twitter. Analyzing the Electoral Communication of the Confluence During the 2015 Council Election. El Profesional de la Información, 26(5), 871-883. https://doi.org/10.3145/epi.2017.sep.09

Graham, T., Jackson, D. y Broersma, M. (2016). New Platform, Old Habits? Candidates' Use of Twitter During the 2010 British and Dutch General Election Campaigns. New Media \& Society, 18(5), 765-783. https://doi.org/10.1177/1461444814546728

Guerrero Solé, F. y Mas Manchón, L. (2017). Estructura de los tweets políticos durante las campañas electorales de 2015 y 2016 en España. El profesional de la información, 26(5), 805-815.

Jungherr, A. (2016). Twitter Use in Election Campaigns: A Systematic Literature Review. Journal of Information Technology \& Politics, 13(1), 72-91. https://doi.org/10.1080/19331681.2015.1132401

Kreiss, D. y Mcgregor, S. C. (2018). Technology Firms Shape Political Communication: The Work of Microsoft, Facebook, Twitter, and Google With Campaigns During the 2016 U.S. Presidential Cycle. Political Communication, 35(2), 155-177. https://doi.org/10.1080/10584609.2017.1364814

Longhi, J. (2013). Essai de caractérisation du tweet politique. L'information grammaticale, Peeters Publishers (13), 25-32.

López- Londoño, L. (2018). La campaña a la Alcaldía de Manizales (Colombia) en Twitter: Un caso para comprender la interacción en línea entre candidatos y ciudadanos. Palabra Clave, 21 (3), 798-830. https://doi.org/10.5294/pacla.2018.21.3.7

Orhuela, J. (2011). Mundo Twitter. Alienta.

Ramos-Serrano, M. (2018). "Follow the Closing of the Campaign on Streaming": The Use of Twitter by Spanish Political Parties During the 2014 European Elections. New Media \& Society, 20(1), 122-140. https://doi.org/10.1177/1461444816660730

Paveau, M.-A. (2012). Genre de discours et technologie discursive. Tweet, twittécriture et twittérature. https://hal.archives-ouvertes.fr/hal-00824817/document\#page=1\&zoom=au to, $-158,848$.

Rieder, B. S., N. (2012). Pluralisme et infomédiation sociale de l'actualité: le cas de Twitter. Réseaux, 176(6), 105-139.

Rodríguez, A. y Ureña, D. (2011). Diez razones para el uso de Twitter como herramienta en la comunicación política y electoral. Comunicación y Pluralismo (10), 89-116.

Roginsky, S. y Cock, L. B. D. (2015). Faire campagne sur Twitter. Modalités d'énonciation et mises en récit des candidats à l'élection européenne. Les Cahiers du numérique, 11(4), 119-144. 
Severo, M. y Lamarche-Perrin, R. (2018). L'analyse des opinions politiques sur Twitter: Défis et opportunités d'une approche multi-échelle. Revue française de sociologie, 59, 505-532. https:// hal.archives-ouvertes.fr/hal-02188391/document

Schroder, K. (2014). Realidades discursivas. La comunicación y los medios metodologías de investigación cualitativa y cuantitativa. Fondo de Cultura Económica.

Slimovich, A. (2016). Política y redes sociales en Argentina. El caso de los candidatos presidenciales de 2011 en Twitter. Signo y Pensamiento, XXXV, 86-100. http://www.scielo.org.co/pdf/signo/ v35n68/v35n68a05.pdf

Stier, S., Bleier, A., Lietz, H. y Strohmaier, M. (2018). Election Campaigning on Social Media: Politicians, Audiences, and the Mediation of Political Communication on Facebook and Twitter. Political Communication, 35(1), 50-74. https://doi.org/10.1080/10584609.2017.1334728

Vallejo, A. (2018). Comunicar emociones en el discurso metapolítico de twitter: El caso de \#MADURO versus@NICOLASMADURO. Observatorio (OBS*),12(3),175-194.

Welp, Y., Freidenberg, F. y Capra, P. (2018). Nuevos medios, vieja política en las elecciones ecuatorianas de 2014. Revista Mexicana de Sociología, 80(4), 827-853.

Zugasti, R. y Sabés, F. (2015). Los issues de los candidatos en Twitter durante la campaña de las elecciones generales de 2011 . Zer: Revista de Estudios de Comunicación, 20(38), 161 178. https:// ojs.ehu.eus/index.php/Zer/article/view/14792 
